\title{
Method transfer across domains and disciplines: enriching universal access development
}

\author{
Christian Stary $\cdot$ Stefan Cronholm
}

Published online: 10 October 2013

(C) Springer-Verlag Berlin Heidelberg 2013

Universal access development procedures refer to the systematic effort to proactively apply principles and tools applicable to universal design, in order to construct accessible and usable interactive systems in various domains. They rely on principles, methods, and tools from accessibility engineering, usability engineering, and user experience [1]. Developments are likely to differ according to sector- or domain-specific properties, even when applying a certain set of methods for different cases (cf. [2]). Reflecting existing developments reveal not only userspecific, but rather method-related transfer elements, as shown by Newell for accessibility engineering:

- Parallels between able-bodied people using technology for particular situations and disabled people using standard interfaces (ordinary and extra-ordinary $\mathrm{HCI}$ ).

- Taking into account the diversity and the rapidly changing characteristics of aging people (design for dynamic diversity).

- An expansion of the methodology of user-centered design to address older and disabled people (usersensitive inclusive design).

- The use of techniques traditionally taught in art school and professional theater both for requirements gathering and for awareness rising (re-contextualization of techniques).

\section{Stary $(\bowtie)$}

Department of Business Information Systems, Communications Engineering, Johannes Kepler University Linz, Linz, Austria e-mail: Christian.Stary@jku.at

\section{S. Cronholm}

School of Business and IT, University of Borås, Borås, Sweden e-mail: Stefan.Cronholm@hb.se
Method transfer concerns the selection and informed application of a design or evaluation method in a specific development context that might have not been addressed so far when applying this method, but could qualify for the development situation at hand. Universal design can be understood as a body of knowledge to support development sensitive to users, domains, and other contextual factors. Given such a case-sensitive appropriation (process), method transfer facilitates

- Taking into account different concerns and stakeholders, as either responsibles, facilitators, actors, developers, or users.

- Mapping the richness of context of use, as given by sector-specific scenarios, emerging technologies for universal use, such as table tops, and culture-specific work environments in an increasingly networked world.

- Bridging the gap between accessibility and usability engineering in the context of user experience.

- Making use of previous development experiences in a structured form, as transfer activities range from adaptation to further development.

The thematic scope of this special issue concerns transfer concepts, techniques, and tools with a view to improving accessibility for a variety of users in different contexts of use. It not only relates to software organization and development but also to task-specific user support, provided that it contributes to improving and widening the apparatus for universal access. As the transfer of methods seems to be related rather to a situation-sensitive procedure than to a (pre-) defined structured set of method attributes and prescribed procedures, one should understand the nature and how to work with adopted procedures.

The issue's intention is to structure and foster the discussion about concepts and experiences when transferring 
methods across domains or sectors. The contributions contain latest research work on the design and implementation of method transfer approaches between different application domains, scenarios of use, contexts, and sectors. Most of the work has been performed in the context of the European COST project TwinTide [3], bringing together researchers revisiting learning practices from the perspective of transferring knowledge from a development situation to another.

The concept of method transfer is revisited from several angels since the authors apply different perspectives and have a background in different research disciplines such as human-computer interaction or information systems. Hence, this issue sets the stage for building method capacity based on situation information. It reveals both conceptual elaborations and concrete appropriation procedures while taking into account existing development traditions.

The requirement for method transfer stems from the growing impact of the fusion of emerging technologies and from the different dimensions of diversity that are intrinsic to application development. Situation dimensions become evident when considering the broad range of user characteristics, the changing nature of human activities, the variety of contexts of use, the increasing diversification of information, knowledge sources and e-services, and the proliferation of technology platforms. All invited authors focus on the increasing design challenges arising from the developments listed, and how they can be handled by learning from specific cases or re-contextualizing methods supporting design or evaluation.

The first contribution entitled "Guiding Situated Method Transfer in Design and Evaluation: Exploring Concepts, Activities and Process" introduces the concept of situated method transfer in design and evaluation. Stefan Cronholm et al. explore transfer activities and develop a process structure aiming toward behavioral guidance when knowledge about method application is transferred from an experienced or documented development situation to the current one. The authors reflect the variety of methods for designing and evaluating interactive socio-technical systems. Taking into account their specificity with respect to development purposes and the observed diversification of interactive systems methods in an effective and efficient way has become decisive for development.

The authors propose a situation framework for learning from method applications in the context of usability of accessibility cases. It lays ground to informed procedures for using existing knowledge on the rationale of method application, domain-specific factors, related assumptions and constraints. Figure 1 shows the frame of reference that can be used for capturing the essence of transfer situations. It provides information on the situation of applying a certain method, i.e., the situation before transfer (left side), and on the situation affected by this knowledge, i.e., the situation after transfer (right side). Each situation is detailed in terms of both the concerned technique or method, and its characteristic (set of) items when in use, constituting the body of knowledge of a method or technique. These items can either be structural, such as roles, or behavioral, such as a sequence of step to be followed when applying a method. One of the items should refer to sector or domain in each situation. The transfer itself is described by the type of activities that are set when moving from one development situation to another.

Cronholm et al. introduce a generic procedure that can be used for informed decision making in development projects with respect to methods. The findings have been based on the contributions of Working Group 2 of the TwinTide project involving a variety of experts in accessibility, usability, and user experience engineering. Finally, the authors introduce some guidelines that should support developers when identifying transfer-relevant items with respect to development situations involving methodological knowledge.

In the subsequent contribution by Carmelo Ardito et al. entitled "On the Transferability of a Meta-Design Model Supporting End-User Development", the transferability of a meta-design model supporting end-user development (EUD) is addressed. The authors show the transfer of such a model recognizing several key elements describing the development context. It is part of structuring previous development experiences in a systematic way. The authors report on experiences of transferring a model that aims at supporting system design by different stakeholders, including domain experts and end users, who want to actively contribute to develop systems better tailored to their end users. Such stakeholders have very different skills and needs; thus, in accordance with universal access principles, the model prescribes that they are provided with interactive environments suitable to them, called Software Shaping Workshops (SSWs), through which stakeholders shape software artifacts. The model capturing this metadesign approach is called SSW model. Key elements of the model have been identified to make model transfer easier. As shown in Fig. 2, supporting participatory creativity was the key for evolving the SSW model. The model key elements facilitated the evolution toward the Hive-Mind Space (HMS) model, both from a structural and from a behavioral perspective.

Several sectors have been addressed. In particular, the SSW model has been applied to healthcare and educational games, where the participation of domain experts to the design is especially relevant, whereas the HMS model has been utilized for home energy management, where end users play the major role and better support for personal 
Fig. 1 Frame of reference describing situated transfer of methods
Generic model for method transfer

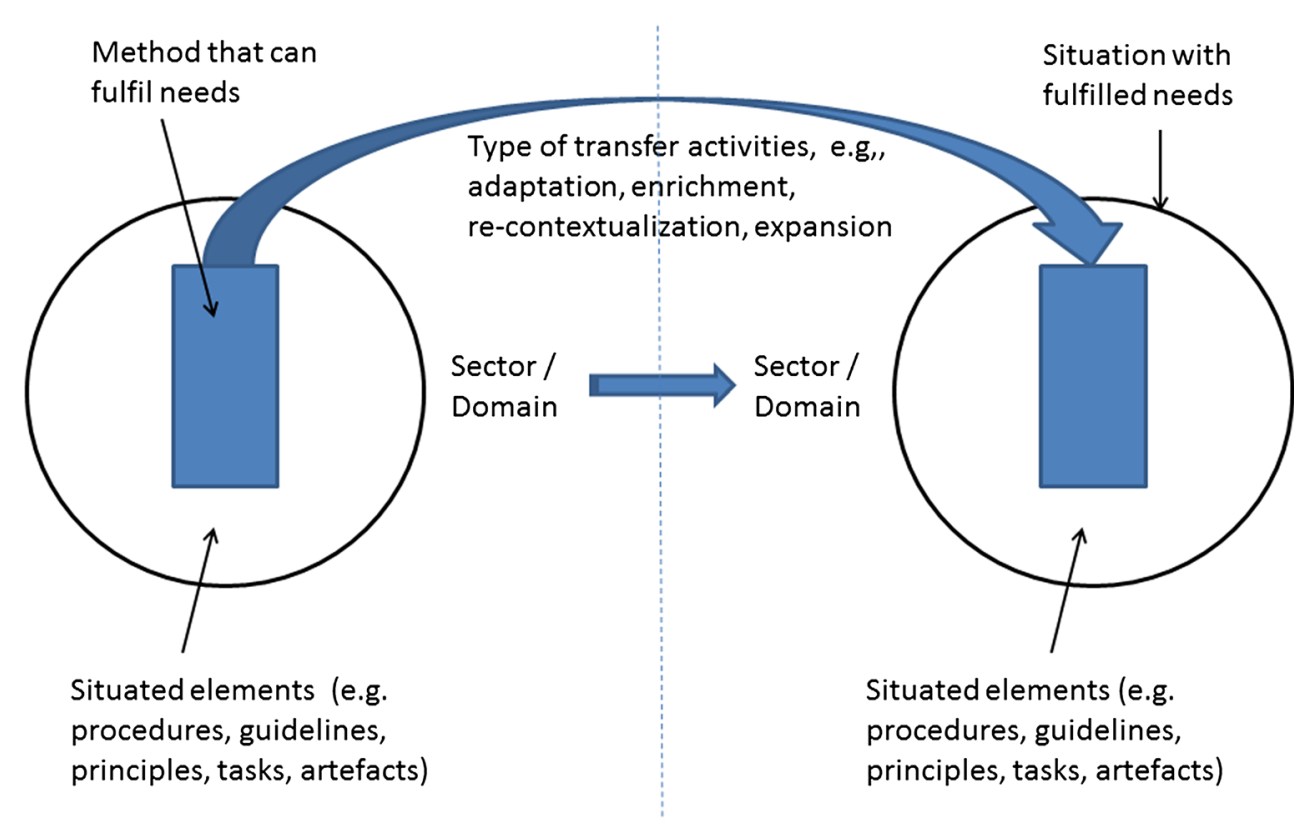

Fig. 2 Transfer through evolving a meta-design model toward participatory creativity
Transfer of a Meta-design Model for End-User Development

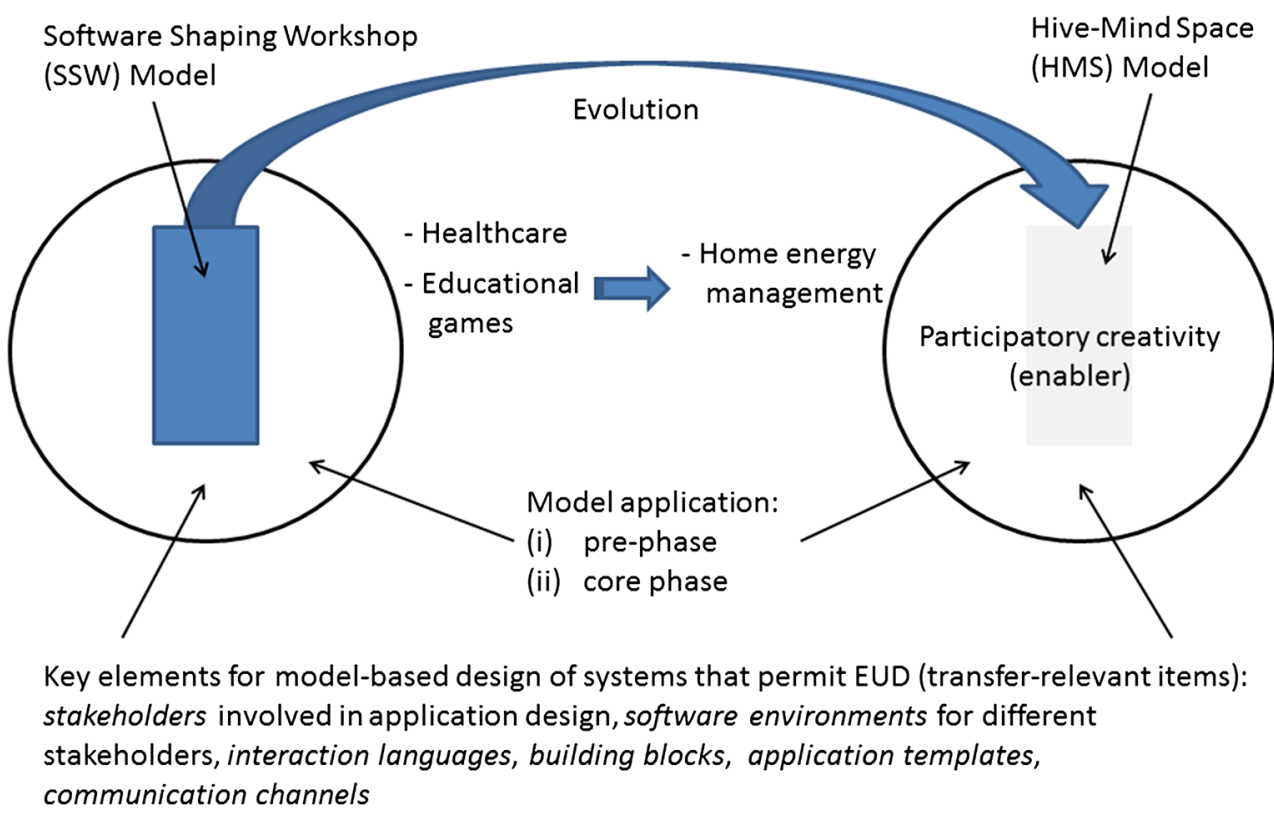

creativity has to be provided. Consequently, the evolution has also enriched application domains not being considered so far by the approach. Informed design has been enabled by the rich set of situational parameters gained from implementing the SSW model.

In her contribution entitled "Identifying and Explicating Knowledge on Method Transfer: A Sectorial System of Innovation Approach", Ebba Hvannberg applies a sectorial system of innovation approach serving as a framework when identifying and explicating knowledge on method transfer. The study reveals from two case studies that the interaction within the sector of crisis management is as important as the interaction with the dimensions of methodological developments. Hence, a frame of reference for a sector facilitates handling the various aspects of method transfer, without restricting the type of methods being handled. It refers to knowledge and technologies, actors and networks, and institutions, as building blocks and 
Fig. 3 Utilizing a sectorial system of innovation for embodying usability testing and heuristic evaluation
Identifying and Explicating Knowledge on Method Transfer:

A Sectorial System of Innovation Approach

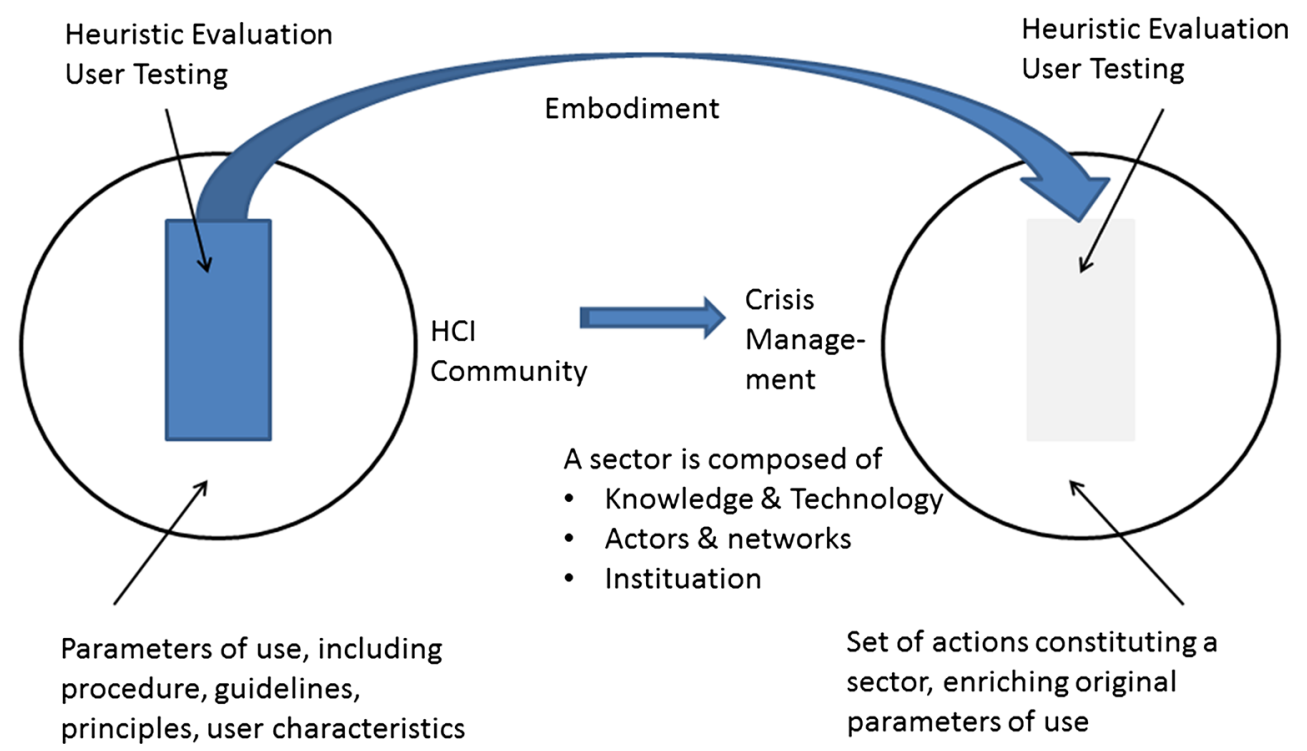

evolutionary theory and actions of innovation as constituents of the transformation process.

The proposed sectorial approach has been applied to evaluation methods, namely heuristic evaluation and user testing. Both methods could be further developed in conformance with the facilities and capabilities of the researchers involved in the sectorial framework of innovation for crisis management. Figure 3 reflects the situation affected by the transfer, identifying the 2 techniques as concerned subjects, including the existing body of knowledge on the HCI community. It has been embodied into each component of the innovation framework for crisis management, tuning it to domain-specific application.

Mark Springett et al. in their contribution "Integrating the Strengths of Cognitive Emotion Models with Traditional HCI Interaction Analysis Tools", deal with integrating the strengths of cognitive emotion models with traditional HCI interaction analysis tools. Their aim is to transfer the strengths of interaction models to analysis of affect critical systems. They start out identifying key concepts from cognitive models of emotion and cognitive models of interaction as given by the HCI literature. In particular, they look at concepts from Scherer's appraisal model and stimulation evaluation checks and Coulson's framework of emotion contexts. This knowledge is transferred by integrating it into Norman's cycle of displaybased action, as it has been applied for interaction analysis in human-computer interaction-see also Fig. 4.

The approach takes into account the recent shift of emphasis to user experience, which requires enriching purely cognition-based approaches to design with affective experiences such as happiness, joy, and surprise. The proposed intertwining of Norman's cognition-based action cycle with models based on appraisal theories should allow shifting the focus toward understanding the causes and effects of feelings arising from interacting with digital artifacts, as indicated by several case studies with older users who are new to digital technology, and e-shoppers.

The subsequent contribution, "Quantitative support for UX methods identification: how can Multiple Criteria Decision Making help?" by Paulo Melo and Luísa Jorge, also impacts design and evaluation, in particular, when making decisions. In search for quantitative support for UX methods identification, multiple criteria decision making is inspected. The authors develop an application scenario on how to apply quantitative multiple criteria decision-making (MCDM) approaches to certain aspects of user experience (UX) design and evaluation. They target the identification of relevant methods in order to proceed with development activities.

In order to reflect strengths and weaknesses of methods, not only the context of use, i.e., the situation needs to be identified, but also characteristic items of the methods need to be specified. Consequently, the proposed MCDM procedure starts out with consensus development on a set of parameters relevant to the methods and the development situation-see also Fig. 5. Then, a model for quantitative matching can be applied corresponding to the transfer process. MCDM methods can be applied to support the informed selection of (UX) methods. Transfer in that context can be considered as configuration of method sets for a particular development context. 
Fig. 4 Enriching interaction analysis by emotion model elements
Integrating the Strength of Cognitive Emotion Models with Traditional $\mathrm{HCl}$ Interaction Analysis Tools

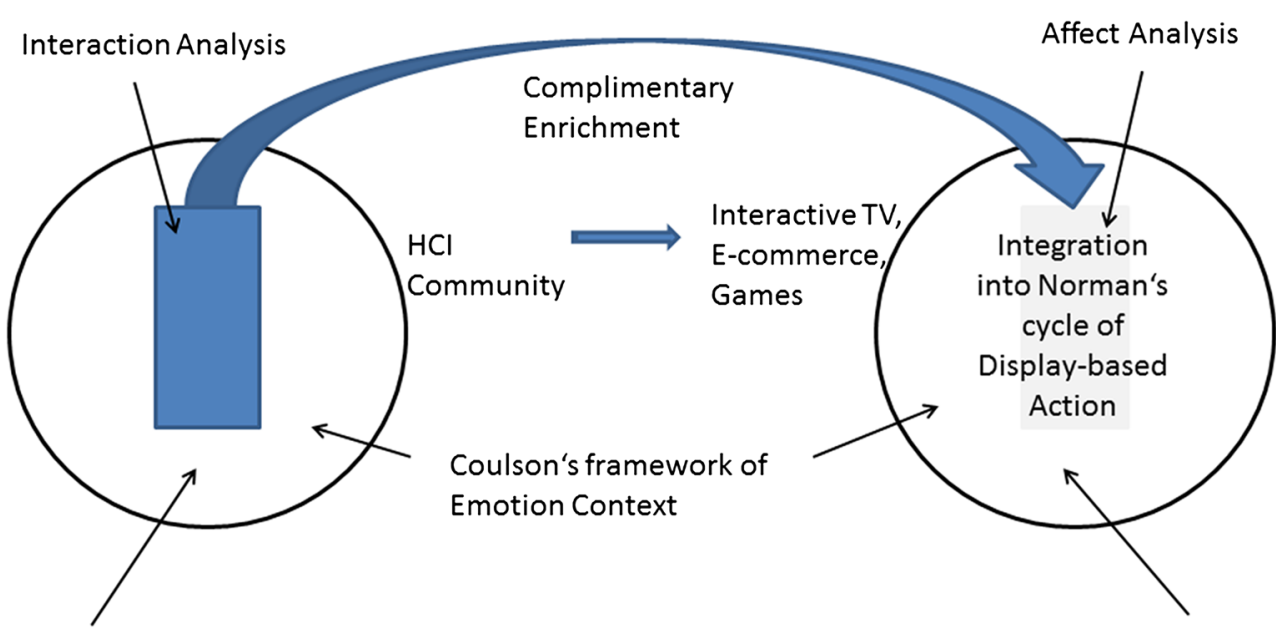

Key concepts of cognitive models of emotion stemming from Scherer's Appraisal Model and Stimulation Evaluation Check

Fig. 5 Informed method set configuration

\section{Quantitative Support for UX Methods Identification: How can Multiple Criteria Decision Making Help?}

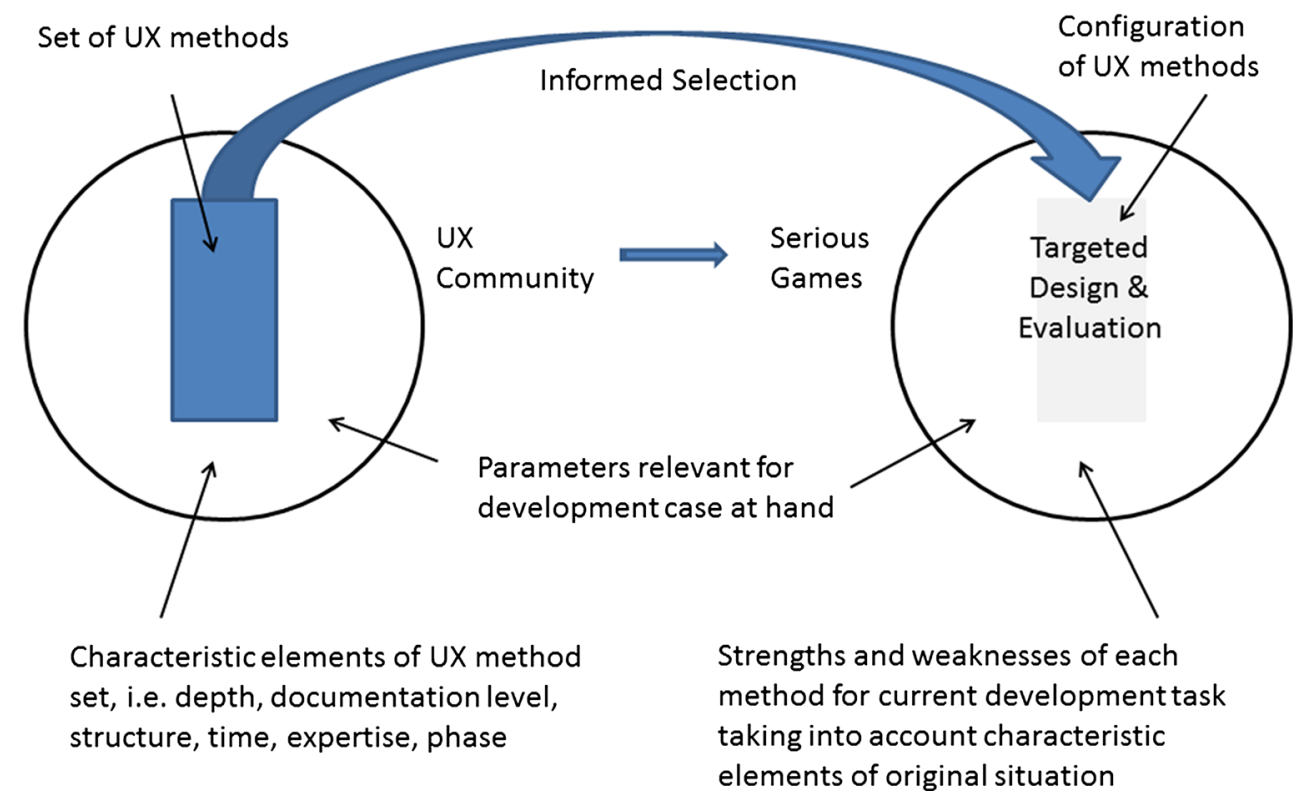

Multi-criteria decision making (MCDM) is also addressed by Bojan Srdjevic et al., in the paper "Virtually combining the analytic hierarchy process and voting methods in order to make group decisions" which proposes combining the analytic hierarchy process and social choice theory (SCT). The authors support group decision making in the course of design. MCDM is represented by the utility approach analytic hierarchy process (AHP), as it can be considered most popular in group decision support. SCT enriches the AHP with a voting system. It can efficiently be combined with AHP in various group decision contexts (Fig. 6).

Group decision-making processes can occur in the field of human-computer interaction using only AHP, either seeking consensus or merely aggregating preferences. As the discourse seems to be important due to the variety of decision makers for universally accessible systems, SCT's preferential and non-preferential voting systems need to be 
Fig. 6 Combining analytical hierarchical process with social theory for informed design decisions

\section{Virtually Combining the Analytic Hierarchy Process and Voting Methods in Order to Make Group Decisions}

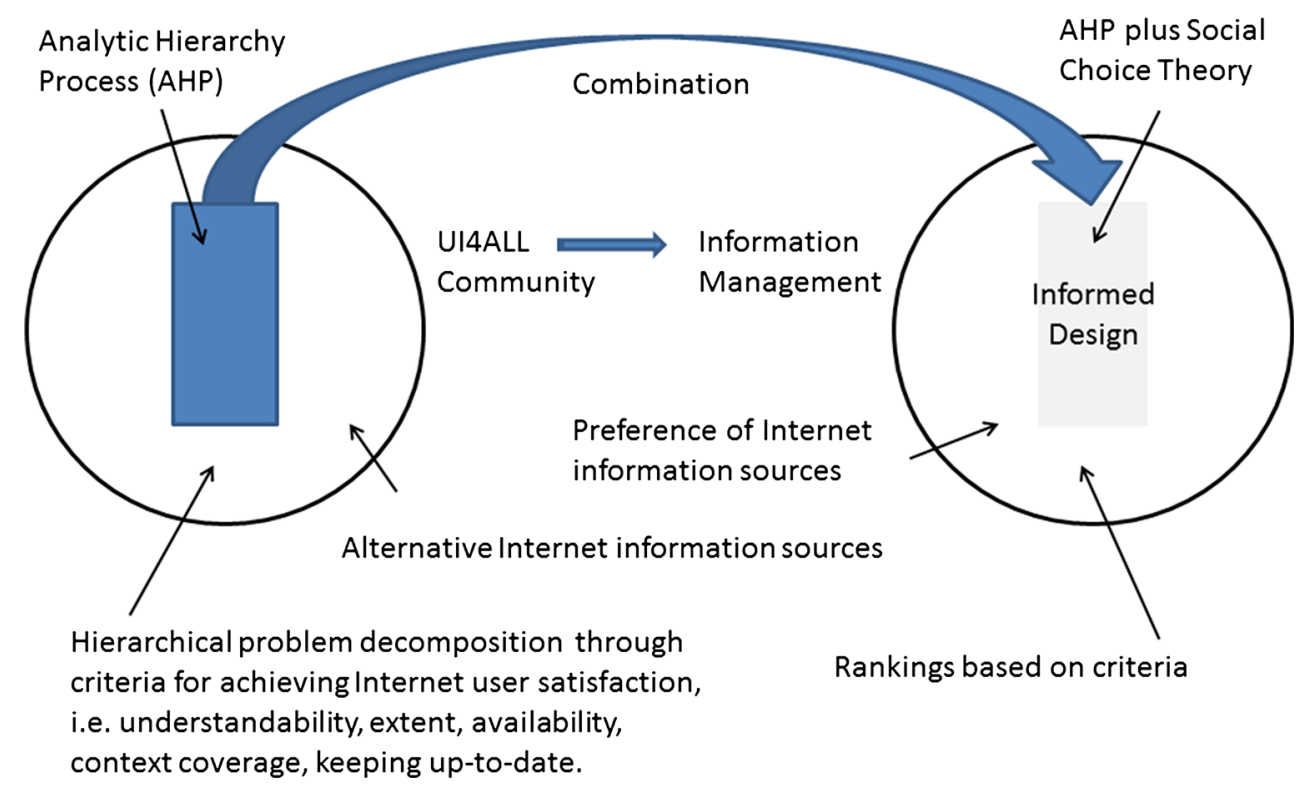

integrated into decision making. Hence, the authors propose to apply AHP in the first stage to obtain weights of the alternatives and, in the second stage, to use AHP's cardinal information, interpret it as ordinal, and use it for the final SCT voting.

The case study reported concerns the utility of sources of information for search tasks. The results reveal plausible decisions, as the decision makers were expected to approve according to their (academic) background.

It should become evident from the contributions outlined above, that transparent method characteristics and contextof-use parameters referring to the development situation can facilitate the transfer of methodological knowledge. It is the reflected situation-sensitive match of parameters that lead to informed design or evaluation decisions. As the starting point of transfer has been dedicated communities, in some cases, namely UI4ALL, UX, and HCI, it could be argued that there is not much documentation of sectorspecific experiences of method applications.
The editors would like to thank all contributors, TwinTide members, the reviewers, and Matthias Neubauer in doing their best making possible this special issue on an essential learning issues aspect of universal design. We are convinced the findings will inspire situated developments and guide further work on the appropriation of interaction know-how.

\section{References}

1. Stephanidis, C. (ed.). The Universal Access Handbook. Taylor \& Francis, London (2009)

2. Newell, A.F. Design and the digital divide: insights from 40 years in computer support for older and disabled people. Synthesis lectures on assistive, rehabilitative, and health-preserving technologies, Morgan \& Claypool (2011)

3. TwinTide. Towards integration of trans-sectorial IT-design and evaluation, cost action IC0904, 2009-2013, European Union Framework Programme 7, www.TwinTide.org (2013) 